\title{
Professor K. G. Ramanathan (1920-1992)
}

by

\author{
S. Raghavan (Bombay)
}

Professor Kollagunta Gopalaiyer Ramanathan who passed away in Bombay on 10 May, 1992, at the age of 72, weighed down during his last few years with serious illness involving cerebral surgery coupled with Parkinson's disease after his retirement in December 1985 from the Tata Institute of Fundamental Research, was a front-rank mathematician of international reputation with distinguished and well-recognized achievements in number theory and especially the analytic and arithmetic theory of quadratic forms over involutorial division algebras. He had contributed in no small measure to the emergence of a new generation of high-calibre mathematicians as well as to the general development of mathematical research and teaching in India.

He was born on 13 November 1920 in Hyderabad in South India. After getting his B.A. degree in Mathematics from Osmania University and M.A. degree from the University of Madras, he worked for some time as a research scholar in the latter. In Madras he came into contact with a genial and inspiring Rev. Fr. Racine as well as with eminent mathematicians like Professors Vaidyanathaswamy and Vijayaraghavan. He also taught for a while, after which he went to Princeton for further study at the Institute for Advanced Study, as assistant to Professor Hermann Weyl. There he came under the influence of the great mathematician Professor Carl L. Siegel, which was much reflected in his subsequent career. Soon after obtaining his Ph.D. degree from Princeton University with Professor Emil Artin for his guide, he returned to India to team up with Professor K. Chandrasekharan at the Tata Institute of Fundamental Research, Bombay, in 1951. With extraordinary dedication and zeal, he joined Professor Chandrasekharan in the magnificent efforts for the steady evolution of the School of Mathematics into an enviable centre for mathematical research with excellence of international level. With his remarkable expertise and all-consuming passion for number theory, he built up the number theory school at TIFR. His polished and meticulously delivered lectures reinforced by informal discussions 
unfolded for many a budding scholar enthralling vistas of the exciting mathematical world of Fermat, Euler, Lagrange, Gauss, Abel, Jacobi, Dirichlet, Kummer, Galois, Eisenstein, Kronecker, Riemann, Dedekind, Minkowski, Siegel, Hilbert, Hecke, Artin, Weil, Chevalley and so on. His abiding enthusiasm for the propagation of good mathematics and the spread of wholesome mathematical culture has been much instrumental in the moulding and flowering of several fine mathematicians and in the betterment of teaching and pursuit of research in Mathematics in many of our universities.

The earlier papers of Professor Ramanathan deal, for the most part, with congruence properties of some arithmetical functions, Ramanujan's trigonometric sums and certain identities of Ramanujan type. Following Siegel's famous work on quadratic forms and P. Humbert's reduction theory, he studied in two of his papers ([12] and [14]) the properties of unit groups of quadratic and hermitian forms over algebraic number fields, such as their finite generation or finiteness of their covolume. In [20], he has used a general formula of Siegel concerning lattice points in symmetric bounded convex euclidean domains to obtain a formula for the discriminant of a division algebra yielding, as a nice consequence, the Hasse-Brauer local-global splitting theorem for the case of quaternion algebras over the rationals.

In an ensuing series of papers, he made a systematic study of the equivalence of and representation by quadratic forms over division algebras with involution and of the unit groups and theta series associated with such quadratic forms, as a prelude to an analytic theory for this general setup on the lines of Siegel's fundamental papers on the analytic theory of quadratic forms (the famous Acta Mathematica papers of Weil in this context appeared later, during 1964-65). Using the foregoing results of his own along with certain methods of Siegel and some theorems due to Selberg and Borel, he solved in his important paper [23] the problem of constructing infinitely many classes of mutually incommensurable discrete groups of the first kind in classical semisimple groups. This was followed by a beautiful paper of his ([24]) wherein he settled the question of maximality of discrete subgroups of arithmetically defined classical groups, generalising certain results of Hecke and Maass. He has also interesting (joint) work establishing the "dense" nature of the set of values at integral algebraic arguments, of "irrational indefinite" quadratic forms representing zero non-trivially (over an algebraic number field), generalising an earlier result of A. Oppenheim, whose well-known conjecture on the values of irrational indefinite quadratic forms at integral arguments was settled only recently by Margulis, using various ideas and techniques from ergodic theory, Lie groups, algebraic groups etc.

For several years, Professor Ramanathan had been actively interested in the study of published and unpublished work of Srinivasa Ramanujan, ex- 
pounding, elucidating and extending Ramanujan's beautiful work on singular values of certain modular functions, Rogers-Ramanujan continued fractions and hypergeometric series. Many mathematicians in the West had made a tremendous advance in respect of many aspects of Ramanujan's unpublished work. In the light of the mathematical prospects so unveiled, he strongly urged many colleagues in India to take seriously to this fascinating domain, even if such activity might be cold-shouldered by "peers" from within. Actually, during the last few months of his life when his right arm was virtually disabled due to Parkinson's disease, he continued to forge ahead in preparing a "monograph on continued fractions" with their two aspects - "one relating to the hypergeometric series and the other related to basic hypergeometric series".

Realising the need to establish a good school in Applications of Mathematics, he mooted, in 1975, the idea of a joint TIFR-IISc Programme to be operated at Bangalore on the campus of the Indian Institute of Science. This Programme has now come of age, with the emergence of a viable group of competent mathematicians specialising in differential equations and numerical analysis, thanks to his efforts and vision.

Although quite attached to his base in Bombay throughout his 34-year tenure with the TIFR, Professor Ramanathan visited many centres of learning, on research and teaching assignments - the Institute for Advanced Study, Princeton, the Mathematisches Institut der Universität Göttingen, the University of Missouri at St. Louis, the University of Alberta at Edmonton etc. He has around 50 research publications to his credit. He was a Fellow of the Indian National Science Academy and of the Indian Academy of Sciences and a Founder Fellow of the Maharashtra Academy of Sciences. He had served as President of the Indian Mathematical Society and as Life President of the Bombay Mathematical Colloquium. As the Editor of the Journal of the Indian Mathematical Society for more than 10 years, he scrupulously maintained a high standard for that journal. He was a member of the Editorial Board for Acta Arithmetica for nearly three decades. He was a recipient of many national awards - the Shanti Swarup Bhatnagar Prize, the Jawaharlal Nehru Fellowship, the Homi Bhabha Medal (from INSA) and the Padma Bhushan.

His interests in English, Telugu and Tamil literature with his unfailing knack for pulling out apt quotations were just as remarkable as his erudition in music. A good conversationalist, he had been heard to remark a couple of times in his later years that the reason for his company being sought was probably that he was considered to be "well-rounded"! However, his occasional quips could have put off a few. He shunned publicity as much as he abhorred those who craved for power and ephemeral glory through the media; those who happened to know him somewhat closely could not have 
failed to note his simplicity and inner humility. For one whose health was indifferent most of the time, he was generally friendly to those who came to him and generous with help when solicited, unmindful of antecedents; he was a firm believer in sharanaagatharakshanam (protecting one coming to seek refuge), in tune with sage Valmiki's aphorism: dosho mahaanathra prapannaanaamarakshane (here lies a great error in not protecting refugeseekers). He was quite against "grabbing" or "snatching" students. He often exhorted fellow number-theorists to pursue their own mathematical objectives diligently without being overawed by glamour or jargon but truly in the anaashrithah karmapalam spirit (not expecting the fruit of action). If he was perhaps sad that his dream of a flourishing number theory school had not entirely been fulfilled, it is for the rest to look for the reasons. In any case, to sum up, his was an illustrious and colourful personality and he will be greatly missed not only by his surviving wife Mrs. Jayalakshmi Ramanathan and two sons Ananth and Mohan to whom he was deeply attached, but also by his countless friends, admirers and former colleagues.

\section{List of papers by K. G. Ramanathan}

1. On Demlo numbers, Math. Student 9 (1941), 112-114.

2. Congruence properties of $\sigma(n)$, the sum of the divisors of $n$, ibid. 11 (1943), 33-35.

3. Multiplicative arithmetic functions, J. Indian Math. Soc. 7 (1943), 111-116.

4. On Ramanujan's trigonometrical sum $C_{m}(n)$, J. Madras Univ. Sect. B 15 (1943), $1-9$.

5. Congruence properties of Ramanujan's function $\tau(n)$, Proc. Indian Acad. Sci. Sect. A 19 (1944), 146-148.

6. Some applications of Ramanujan's trigonometrical sum $C_{m}(n)$, ibid. 20 (1944), $62-69$.

7. Congruence properties of $\sigma_{a}(n)$, Math. Student 13 (1945), 30.

8. Congruence properties of Ramanujan's function $\tau(n)$, II, J. Indian Math. Soc. 9 (1945), 55-59.

9. Congruence properties of $\sigma_{a}(N)$, Proc. Indian Acad. Sci. Sect. A 25 (1947), 314-321.

10. On the product of the elements in a finite Abelian group, J. Indian Math. Soc. 11 (1947), 44-48.

11. Identities and congruences of the Ramanujan type, Canad. J. Math. 2 (1950), 168-178.

12. The theory of units of quadratic and hermitian forms, Amer. J. Math. 73 (1951), $233-255$.

13. Abelian quadratic forms, Canad. J. Math. 4 (1952), 352-368.

14. Units of quadratic forms, Ann. of Math. 56 (1952), 1-10.

15. A note on symplectic complements, J. Indian Math. Soc. 18 (1954), 115-125.

16. The Riemann sphere in matrix spaces, ibid. 19 (1955), 121-125.

17. Quadratic forms over involutorial division algebras, ibid. 20 (1956), 227-257.

18. Units of fixed points in involutorial algebras, in: Proc. Internat. Sympos. on Algebraic Number Theory, Science Council of Japan, Tokyo 1956, 103-106. 
19. On orthogonal groups, Nachr. Akad. Wiss. Göttingen Math.-Phys. Kl. II 1957, $113-121$.

20. The zeta function and discriminant of a division algebra, Acta Arith. 5 (1959), $277-288$

21. Quadratic forms over involutorial division algebras II, Math. Ann. 143 (1961), 293-332.

22. Zeta functions of quadratic forms, Acta Arith. 7 (1961), 39-69.

23. Discontinuous groups, Nachr. Akad. Wiss. Göttingen Math.-Phys. Kl. II 1963, 293-323.

24. Discontinuous groups II, ibid. 1964, 145-164.

25. (with S. Raghavan) On a Diophantine inequality concerning quadratic forms, ibid. 1968, 251-262.

26. A converse of a theorem of Siegel, in: Prof. Ananda-Rau Memorial Volume, Publ. Ramanujan Inst. 1, Madras 1969, 291-296.

27. S. Minakshisundaram, J. Indian Math. Soc. 34 (1970), 135-149.

28. (with S. Raghavan) Values of quadratic forms, ibid., 253-257.

29. (with S. Raghavan) Solvability of a Diophantine inequality in algebraic number fields, Acta Arith. 20 (1972), 299-315.

30. On the analytic theory of quadratic forms, ibid. 21 (1972), 423-436.

31. Theory of numbers, J. Sci. Industrial Res. 31 (1972), 459.

32. Srinivasa Ramanujan, Mathematician extraordinary, Science Today, Dec. 1974.

33. C. P. Ramanujam, in: C. P. Ramanujam-A Tribute, Tata Inst. Fund. Res. Stud. Math. 8, Springer, Berlin 1978, 1-7.

34. (with M. V. Subbarao) Some generalizations of Ramanujan's sum, Canad. J. Math. 32 (1980), 1250-1260.

35. Ramanujan and the congruence properties of partitions, Proc. Indian Acad. Sci. Math. Sci. 89 (1980), 133-157.

36. The unpublished manuscripts of Srinivasa Ramanujan, Current Science 50 (1981), 203-210.

37. Remarks on some series considered by Ramanujan, J. Indian Math. Soc. 46 (1982), $107-136$

38. Subramiah Minakshisundaram (1913-1968), Bull. Math. Assoc. India 14 (1982), $27-32$.

39. On Ramanujan's continued fraction, Acta Arith. 43 (1984), 209-226.

40. On the Rogers-Ramanujan continued fraction, Proc. Indian Acad. Sci. Math. Sci. 93 (1984), 67-77.

41. Ramanujan's continued fraction, Indian J. Pure Appl. Math. 16 (1985), 695-724.

42. Srinivasa Ramanujan, 22 December 1887-26 April 1920, J. Indian Math. Soc. 51 (1987), 1-25.

43. Some applications of Kronecker's limit formula, ibid. 52 (1987), 71-89.

44. Ramanujan's Notebooks, J. Indian Inst. Sci. 1987, Ramanujan Special Issue, 25-32.

45. Hypergeometric series and continued fractions, Proc. Indian Acad. Sci. Math. Sci. 97 (1987), 277-296.

46. Generalisations of some theorems of Ramanujan, J. Number Theory 29 (1988), $118-137$.

47. On some theorems stated by Ramanujan, in: Number Theory and Related Topics, Tata Inst. Fund. Res. Stud. Math. 12, Oxford Univ. Press, 1989, 151-160.

48. Ramanujan's modular equations, Acta Arith. 53 (1990), 403-420. 


\section{Books}

1. On the Algebraic Theory of Fields, Tata Inst. Fund. Res. Lectures on Math. 5, Tata Inst. Fund. Res., 1954.

2. (with M. Pavaman Murthy, C. S. Seshadri, U. Shukla and R. Sridharan) Galois Theory, Math. Pamphlets 3, Tata Inst. Fund. Res., 1965.

SCHOOL OF MATHEMATICS

TATA INSTITUTE OF FUNDAMENTAL RESEARCH

HOMI BHABHA ROAD

BOMBAY 400005, INDIA

Received on 28.9.1992

and in revised form on 22.12.1992 